\title{
Optical and electronic properties of bismuth-implanted glasses
}

\author{
M. A. Hughes, ${ }^{a}$ Y. Federenko, ${ }^{a}$ T. H. Lee, ${ }^{c}$ J. Yao, ${ }^{b}$ B. Gholipour, ${ }^{b}$ R. M. Gwilliam, ${ }^{a}$ K. P. \\ Homewood, ${ }^{a}$ D. W. Hewak, ${ }^{b}$ S. R. Elliott, ${ }^{c}$ and R. J. Curry ${ }^{a}$ \\ aAdvanced Technology Institute, Department of Electronic Engineering, University of Surrey, \\ Guildford, GU2 7XH, United Kingdom; ' ${ }^{b}$ ptoelectronics Research Centre, University of \\ Southampton, Southampton SO17 1BJ, United Kingdom; ' Department of Chemistry, University of \\ Cambridge, Lensfield Road, Cambridge, CB2 1EW, United Kingdom
}

\begin{abstract}
Photoluminescence (PL) and excitation spectra of Bi melt-doped oxide and chalcogenide glasses are very similar, indicating the same $\mathrm{Bi}$ center is present. When implanted with $\mathrm{Bi}$, chalcogenide, phosphate and silica glasses, and $\mathrm{BaF}_{2}$ crystals, all display characteristically different PL spectra to when Bi is incorporated by melt-doping. This indicates that ion implantation is able to generate $\mathrm{Bi}$ centers which are not present in samples whose dopants are introduced during melting. Bi-related PL bands have been observed in glasses with very similar compositions to those in which carrier-type reversal has been observed, indicating that these phenomena are related to the same Bi centers, which we suggest are interstitial $\mathrm{Bi}^{2+}$ and $\mathrm{Bi}$ clusters.
\end{abstract}

Keywords: bismuth, implantation, chalcogenide, photoluminescence

*Electronic mail: m.a.hughes@surrey.ac.uk

\section{Introduction}

Bismuth-doped glasses can give rise to photoluminescence (PL) at wavelengths ranging from $400 \mathrm{~nm}$ [1] to $2500 \mathrm{~nm}$ [2], under variation of the pump wavelength and composition of the host glass. A wide variety of traditional glass hosts containing Bi have been investigated to date, mainly silicates [3-5] and germanates [6-8], but also phosphates [9], borates [10], chalcogenides [11, 12] and chlorides [2]. The origin of the infrared emission from Bi-doped glasses remains controversial, with convincing arguments being made for a variety of different emission centers, including $\mathrm{Bi}^{+}[10], \mathrm{Bi}^{5+}$ $[13,14]$, Bi metal clusters [8], point defects [15] and negatively charged $\mathrm{Bi}_{2}$ dimers [16, 17]. However, there is a general consensus developing that more than one Bi center is responsible for the observed optical activity of the Bi dopant in glass. $\mathrm{Pb}$ doping has also been shown to have very similar absorption and PL behavior to that of Bi in glasses [15]. Broadband Bi-doped fiber lasers operating at wavelengths between 1150 and $1550 \mathrm{~nm}$ [18], with powers up to $20 \mathrm{~W}$ [19] and slope efficiencies of up to $30 \%$ [20], have been reported. A mode-locked Bi-doped fiber laser with 900 fs pulses has also been demonstrated [21]. Bismuth-doped glasses are therefore, potentially, an extremely important class of material for use in broadband lasers and optical amplifiers. One of the main limitations of Bi-doped fiber lasers fabricated so far is the inability to obtain lasing with doping concentrations more than around $0.005 \mathrm{wt} \%$ [22]. This means that the fibers need to be on the order of $100 \mathrm{~m}$ in length, which causes problems of background loss and nonlinearities. Absorption tails located close to lasing wavelengths cause additional losses [23]. The presence of Bi centers not involved in the lasing process could cause concentration quenching and absorption losses. Therefore, an understanding of the nature of the Bi centers, and the ability to control which Bi centers are present, is critical for the ability to increase doping concentration, reduce losses and bring the performance of $\mathrm{Bi}$-doped fiber lasers in line with that of rare-earth-doped fiber lasers.

Chalcogenide glasses are a broad class of increasingly important technological materials used in phase-change memories, solar cells, sensors and non-linear optical devices. They almost invariably display p-type electronic conductivity, and are known to remain p-type when melted with common donor atoms. The ability to reverse the carrier type in these glasses would enable electronic devices to be integrated with other chalcogenide-glass-based devices and may enable the 
fabrication of LEDs and laser diodes that emit at novel wavelengths. $\mathrm{Bi}$ and $\mathrm{Pb}$ are the only known dopants to cause carriertype reversal (CTR) in chalcogenide glasses by melt doping, mainly in germanium chalcogenides. The origin of this carriertype reversal is also disputed. Phillips proposed a model for the microscopic structure of Bi-modified $\mathrm{Ge}_{x} \mathrm{~B}_{1-x}(\mathrm{~B}=\mathrm{S}, \mathrm{Se}$ or Te) glass as $\mathrm{Bi}_{2} \mathrm{~B}_{3}$ clusters with a tetradymite-like structure embedded in a $\mathrm{Ge}_{x} \mathrm{~B}_{1-x}$ matrix [24]. This was based on differential thermal analysis (DTA) [25] and penetration probe [26] measurements which indicated a phase separation in the Bi-modified glasses. A.C. measurements indicate that these clusters may have $n$-type defects situated at excess $\mathrm{S}^{-}$ atoms on cluster surfaces; these defects may be taking part in a single-polaron hopping process [27]. However, on the basis of EXAFS measurements, Elliott et al argued that $\mathrm{Bi}$ is only 3-fold coordinated, and that the glasses were homogeneous without $\mathrm{Bi}_{2} \mathrm{~S}_{3}$ clusters [28], and that the mechanism of CTR is due to the presence of charged $\mathrm{Bi}$ atoms which suppress the concentration of positively charged chalcogen defects at the expense of negatively charged defects. In 1989, a p-n junction based on the Ge-Se-Bi glass system was fabricated [29]. However, since this time, there have not been significant strides in increasing the performance of these p-n junctions, which may be related to the lack of understanding of the physical process that underlies carrier-type reversal in these glasses.

Conventionally, $\mathrm{Bi}$ and $\mathrm{Pb}$ dopants are introduced into the glass melts ('melt-doping'). We define melt-doping as an equilibrium doping method because the dopants are able to react with the glass material, above the glass-transition temperature, $T_{\mathrm{g}}$, for sufficient time for the dopants to achieve their lowest energy bonding configuration. We define nonequilibrium doping as the inclusion of a dopant into the glass matrix below $T_{\mathrm{g}}$. Ion implantation is a precise, nonequilibrium doping technique which is essential to the fabrication of most modern integrated circuits (ICs). It is relevant to Bi-doped glasses because it may allow control over which Bi centers are present in the glass. It is also relevant for the development of high-performance electronic devices based on Bi-doped glasses because it is the most precise doping technique in use today, and it may be possible to reverse the carrier type of chalcogenide glasses by impurity doping under non-equilibrium conditions [30], as has been shown for $\mathrm{Cd}, \mathrm{Al}, \mathrm{Zn}$ and $\mathrm{Mg}$ (non-equilibrium) diffusion-doped $\mathrm{As}_{2} \mathrm{Se}_{2} \mathrm{Te}_{1}$ glass [31-33]. In this work, we report PL from Bi-implanted glasses and crystals. The PL spectra are different to those from similar materials that are melt doped with Bi. We also report PL from Bi-doped chalcogenide glasses which have compositions very close to those in which carrier-type reversal has been reported, and suggest that these phenomena are produced by the same, or a similar, active center. If our hypothesis is correct, it may assist in hastening the elucidation of the origin of these phenomena.

\subsection{Sample preparation}

\section{Experimental}

A gallium lanthanum sulphur oxide (GLSO) sputtering target was prepared by mixing $70 \%$ gallium sulphide, and $30 \%$ lanthanum oxide in a dry-nitrogen purged glove box. The raw materials were melted for 24 hours in dry argon, in 2 inch diameter vitreous carbon crucibles, annealed at the glass-transition temperature and then sliced to form a $3 \mathrm{~mm}$ thick sputter target. We sputtered $100 \mathrm{~nm}$ thick films of GLSO onto $1 \mu \mathrm{m}$ thick thermally oxidized $\mathrm{SiO}_{2}$ on $\mathrm{Si}$ substrates. The RF sputtering power was 60W, with an Ar flow of 15 SCCM. Details of the fabrication of bulk Bi-doped GLSO [12] and SiAlLiO [34] can be found elsewhere. Fused silica glass, Schott NPSK53 phosphate glass, crystalline $\mathrm{CaF}_{2}$ and $\mathrm{BaF}_{2}$ polished bulk samples were purchased commercially. Bismuth ions were implanted using a Danfysik ion implanter at an energy of $190 \mathrm{keV}$. During implantation, the samples were mounted on a carousel holder held at ambient temperature, and the beam current was kept below $1 \mu \mathrm{A} / \mathrm{cm}^{2}$ to avoid beam heating of the targets.

\subsection{Sample characterization}

PL spectra of bulk samples were obtained by exciting with a $808 \mathrm{~nm}$ laser diode, or $514 \mathrm{~nm}$ Ar-ion laser. The emission was dispersed by a Jasco CT-25C monochromator which used a 600 or 1200 lines $/ \mathrm{mm}$ grating. The slit width was $\sim 2.5$ $\mathrm{mm}$, which corresponded to a resolution of $\sim 10 \mathrm{~nm}$. The stray excitation light was blocked with appropriate long-pass filters. Detection was realized with a Hamamatsu H9170 NIR photomultiplier tube (PMT), Newport Si, or InGaS detectors, coupled with standard phase-sensitive detection. All spectral measurements were corrected for the wavelength-dependent response of the measurement system by calculating a correction spectrum $(C(\lambda))$, with $C(\lambda)=I_{\text {cal }}(\lambda) / I_{\text {meas }}(\lambda)$, where $I_{\text {meas }}(\lambda)$ is the luminescence spectrum of an Ushio calibrated white-light source measured by the detection system and $\mathrm{I}_{\text {cal }}(\lambda)$ is the luminescence spectrum of the calibrated white-light source supplied by the manufacturer. Due to their weak emission, PL spectra of implanted samples were taken on a Renishaw 2000 microRaman system incorporating a Si CCD detector array, with a detection range of $400-1100 \mathrm{~nm}$, and 514 or $782 \mathrm{~nm}$ excitation laser lines. A 50x microscope objective was used to focus the excitation onto the implanted sample surface. Several spectra were taken at different positions on the sample 
and then averaged. It was found that the variation in PL intensity between different positions on the same sample was less than 5\%. This technique can therefore be used to compare the relative PL intensity between different implants. We also measured the PL from unimplanted samples to account for any PL that could be coming from the unimplanted film or substrate. These spectra were then subtracted from the PL spectra of the implanted samples. The PL intensity of unimplanted samples was less than around 5\% of that of the implanted samples in the thin-film samples, and less than 25\% in the bulk samples. Spectra were corrected by measuring the broadband PL of a Bi-doped glass with a known spectral luminance. Ripples in some of the PL spectra measured on the Raman system are an artifact caused by the various filters. Differential thermal analysis (DTA) measurements were taken using a Rigaku Thermo Plus TG-DTA. Rutherford backscattering (RBS) measurements were made on a $2 \mathrm{MV}$ Tandetron accelerator using $2 \mathrm{MeV}$ He beams.

\subsection{Melt-doped oxides and chalcogenides}

\section{Results and discussion}

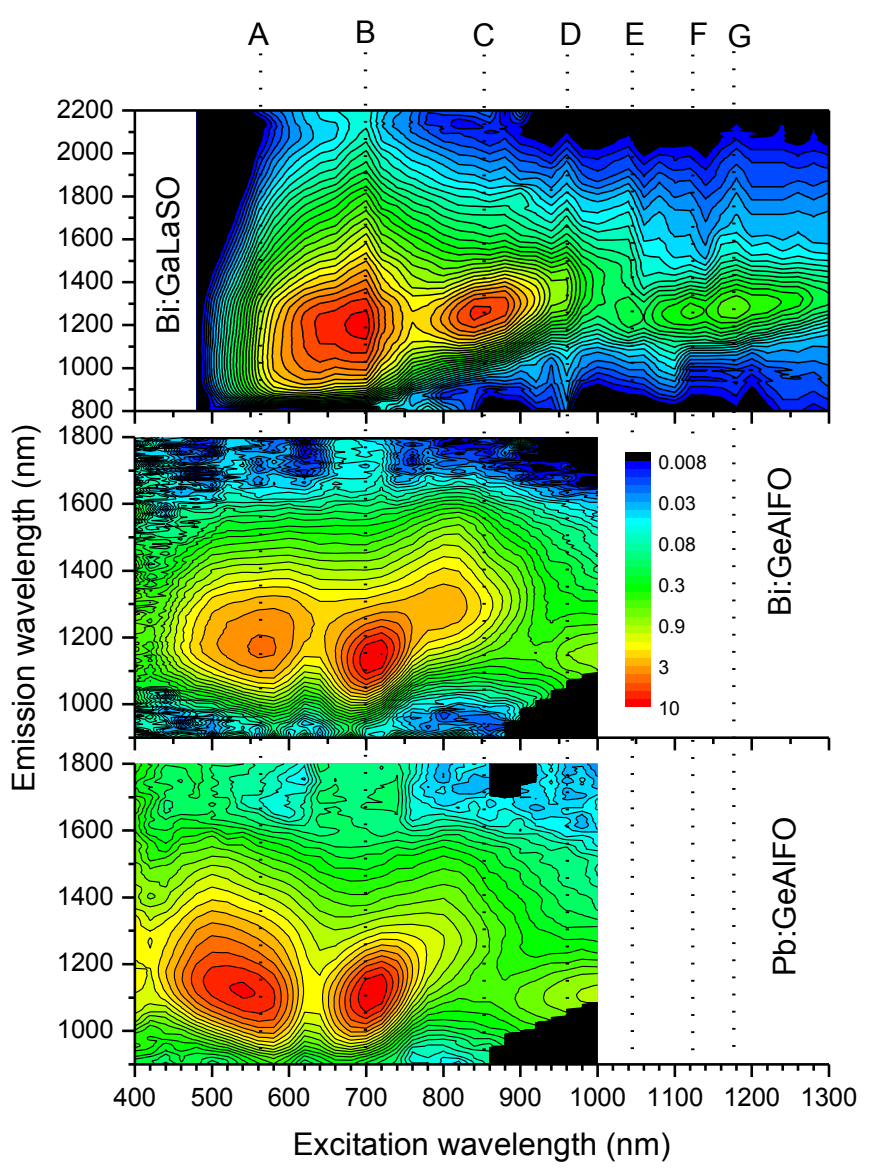

Figure 1 Contour plots of PL emission from Bi-doped GLSO, after [12], and Bi- and Pb-doped germanate bulk glasses, after [15]. The intensity is plotted on a log scale.

Figure 1 shows contour plots, which are comprised of a series of PL spectra over a range of excitation wavelengths, of bulk melt-doped glasses: $\mathrm{Ga}_{28} \mathrm{La}_{12} \mathrm{~S}_{56} \mathrm{O}_{4} \mathrm{Bi}_{0.4}$ (Bi:GLSO), after [12], $\mathrm{Ge}_{28} \mathrm{O}_{56} \mathrm{~F}_{11} \mathrm{Al}_{4} \mathrm{Bi}_{0.6}$ (Bi:GeAlFO), and $\mathrm{Ge}_{28} \mathrm{O}_{56} \mathrm{~F}_{12} \mathrm{~A}_{14} \mathrm{~Pb}_{0.3}$ (Pb:GeAlFO), after [15]. These contour plots can be used to determine the position of absorption and PL bands. Strong absorption/PL bands show up clearly as islands on the contour plot; weaker absorption/PL bands show up as kinks and bends in the contour lines. In Fig 1, we identified seven absorption/PL bands in Bi:GLSO, marked A-G. Band A is rather indistinct in Bi:GLSO, which may be caused by the strong band-edge absorption of GLSO in this region. However, there 
is a clear change in direction of the contour lines at $565 \mathrm{~nm}$, indicating the presence of an absorption/PL band, which lines up almost exactly with the strong band in Bi:GeAlFO. Band D is evident only as a kink in contour lines in Bi:GeAlFO at $960 \mathrm{~nm}$, and is not evident in $\mathrm{Pb}: \mathrm{GeAlFO}$. Much of band $\mathrm{E}$ is missing in the germanate glasses; however, it is not much greater than $1000 \mathrm{~nm}$. We find that the positions of 5 absorption/PL bands are approximately the same in $\mathrm{Bi}$ - and $\mathrm{Pb}$-doped germanate glass and Bi-doped chalcogenide glass, indicating that they are related to essentially the same optical center. Similar absorption and emission properties were also observed in $\mathrm{Bi}$ - and $\mathrm{Pb}$-doped calcium phosphate and sodium silicate glasses [35]. The origin of this similarity is intriguing. Similar NIR PL bands have been reported from $\mathrm{Bi}-, \mathrm{Pb}-, \mathrm{Sb}-$ and Sn-doped germanate glasses [15], and since spin-orbit constants are so dissimilar for these elements, it has been argued that the NIR PL does not originate from dopant centers. We suggest, however, that there may be similarities in the energylevel structure of complex clusters of these elements, which could produce similar NIR PL bands.

\subsection{Ion-implanted optical materials}

Figure 2 shows the PL spectra of Bi-implanted GLSO films along with bulk phosphate and silica glass, and crystalline $\mathrm{CaF}_{2}$ and $\mathrm{BaF}_{2}$, excited at $782 \mathrm{~nm}$. Damage-related optical centers are known to produce PL; however, when implanted with other species such as Ag and V, no PL was observed. This indicates that the observed PL is related to a Bi center, rather than damage caused by the implantation. The Bi-implanted samples all display a PL peak at 800 to $880 \mathrm{~nm}$. Phosphate glass has a PL peak at $800 \mathrm{~nm}$, which is very close to the excitation wavelength. GLSO and silica both have a main PL peak at $820 \mathrm{~nm}$, with GLSO having a shoulder at $920 \mathrm{~nm}$. Crystalline $\mathrm{BaF}_{2}$ has a peak at $845 \mathrm{~nm}$ with evidence of a shoulder at $960 \mathrm{~nm}$. Crystalline $\mathrm{CaF}_{2}$ has a single broad peak at $880 \mathrm{~nm}$. When melt doped with $\mathrm{Bi}$ in bulk and excited at $800 \mathrm{~nm}$, phosphate glass has a broad PL peaking at $1300 \mathrm{~nm}$ [36], silica glass at $1250 \mathrm{~nm}$ [37] and crystalline $\mathrm{BaF}_{2}$ at $1100 \mathrm{~nm}$ [38]. This result indicates that ion implantation is able to generate Bi centers which are not present in samples whose dopants are introduced during melting. This is important for the application of $\mathrm{Bi}$ implantation as a non-equilibrium doping technique because it indicates that the Bi does not achieve its lowest energy bonding configuration as is expected during melt doping. The intensity of the PL varied by over two orders of magnitude between the phosphate and silica glass host.

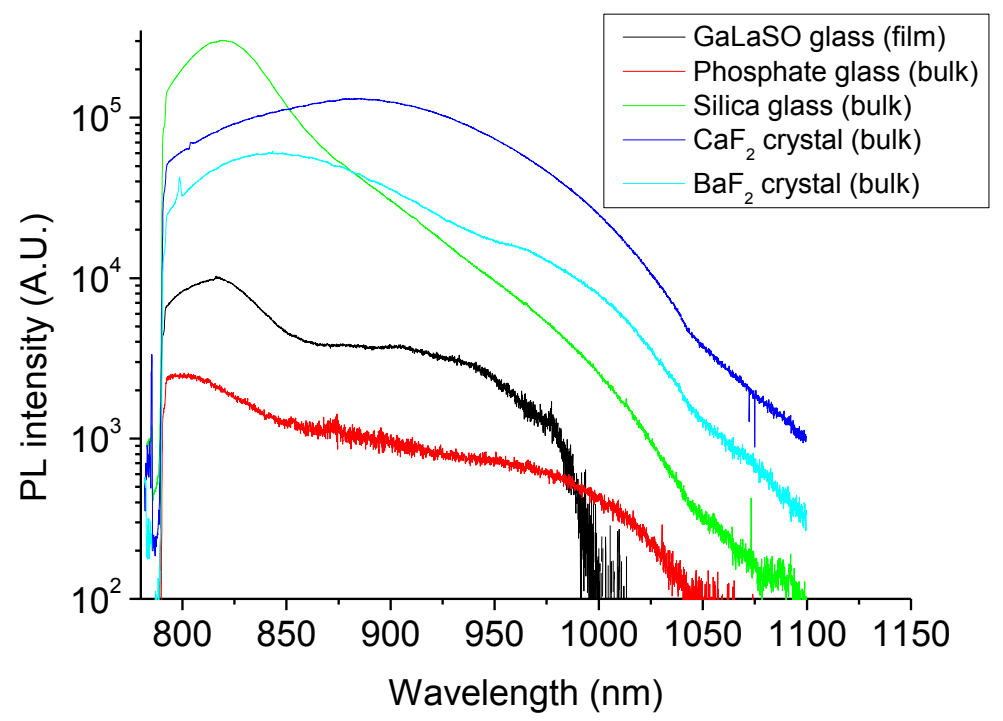

Figure 2 PL spectra of Bi-implanted GLSO thin film, bulk phosphate and silica glass, and crystalline $\mathrm{CaF}_{2}$ and $\mathrm{BaF}_{2}$, at a dose of $3 \times 10^{15}$ ions $/ \mathrm{cm}^{2}$. Excitation was at $782 \mathrm{~nm}$.

Figure 3 shows the PL spectra of Bi-implanted GLSO thin films at various doses, along with bulk Bi-melt-doped GLSO and $\mathrm{Li}_{2} \mathrm{O}-\mathrm{Al}_{2} \mathrm{O}_{3}-\mathrm{SiO}_{2}(\mathrm{LiAlSiO})$ glass, excited at $514 \mathrm{~nm}$. Bulk Bi:GLSO exhibits a PL peak at $950 \mathrm{~nm}$. However, the 
$1 \times 10^{14} \mathrm{ions} / \mathrm{cm}^{2} \mathrm{Bi}$-implanted GLSO has a characteristic red PL band, peaking at $700 \mathrm{~nm}$, which is commonly observed in $\mathrm{Bi}$-doped oxide glasses under green excitation [39]. The spectrum of the Bi:LiAlSiO glass in Figure 3 also has a $700 \mathrm{~nm}$ PL band, which is very similar to that of the $1 \times 10^{14}$ ions $/ \mathrm{cm}^{2}$ Bi-implanted GLSO. Comparing the $1 \times 10^{15}$ ions $/ \mathrm{cm}^{2} \mathrm{Bi}$ implant to bulk Bi:GLSO (which have very similar compositions) indicates that the $1 \times 10^{15} \mathrm{ions} / \mathrm{cm}^{2} \mathrm{Bi}$ implant has the $700 \mathrm{~nm}$ PL band associated with oxide glasses, and the $950 \mathrm{~nm}$ PL band observed in bulk Bi:GLSO, in equal intensities. If we extend our model of oxide glasses, in which red and NIR PL result from $\mathrm{Bi}^{2+}$ and $\mathrm{Bi}_{n}$ clusters, respectively [40], to $\mathrm{Bi}$ GLSO, then implanted $\mathrm{Bi}$ is more likely to be incorporated as $\mathrm{Bi}^{2+}$ than melt-doped $\mathrm{Bi}$. The $700 \mathrm{~nm}$ PL band remains approximately constant for $1 \times 10^{14}$ and $1 \times 10^{15}$ ions $/ \mathrm{cm}^{2} \mathrm{Bi}$ implants, which is similar to the effect discussed earlier for $\mathrm{Bi}: \mathrm{SiMgAlO}$ [39]. For the $1 \times 10^{16}$ ions $/ \mathrm{cm}^{2} \mathrm{Bi}$ implant, the $700 \mathrm{~nm}$ PL band becomes stronger. We were unable to detect any NIR PL from the implanted samples using the standard measurement system that we used for our bulk samples.

During ion implantation, accelerated ions are decelerated by collisions with the nuclei, and electronic clouds, of atoms in the target. After a series of collisions, the implanted ion comes to rest. These collisions result in the displacement of individual atoms, which causes damage to the atomic structure of the target. In silicon IC manufacture, this damage is usually relieved by subsequent annealing above the crystallization temperature. This annealing activates the dopants by allowing them to move from an interstitial site to a lattice site. Similarly to ion-implanted $\mathrm{Si}$, we expect that ions implanted into chalcogenide glasses will initially enter interstitial sites. XPS and NEXAFS measurements of $\mathrm{N}_{2}$-implanted and nitrogen-co-deposited $\mathrm{Ge}_{2} \mathrm{Sb}_{2} \mathrm{Te}_{5}$ amorphous chalcogenide films indicated that implanted $\mathrm{N}$ tended to accumulate in interstitial sites compared to co-deposited $\mathrm{N}_{2}$ [41]. This indicates that $\mathrm{Bi}^{2+}$ associated with Bi-implanted GLSO may be interstitial.

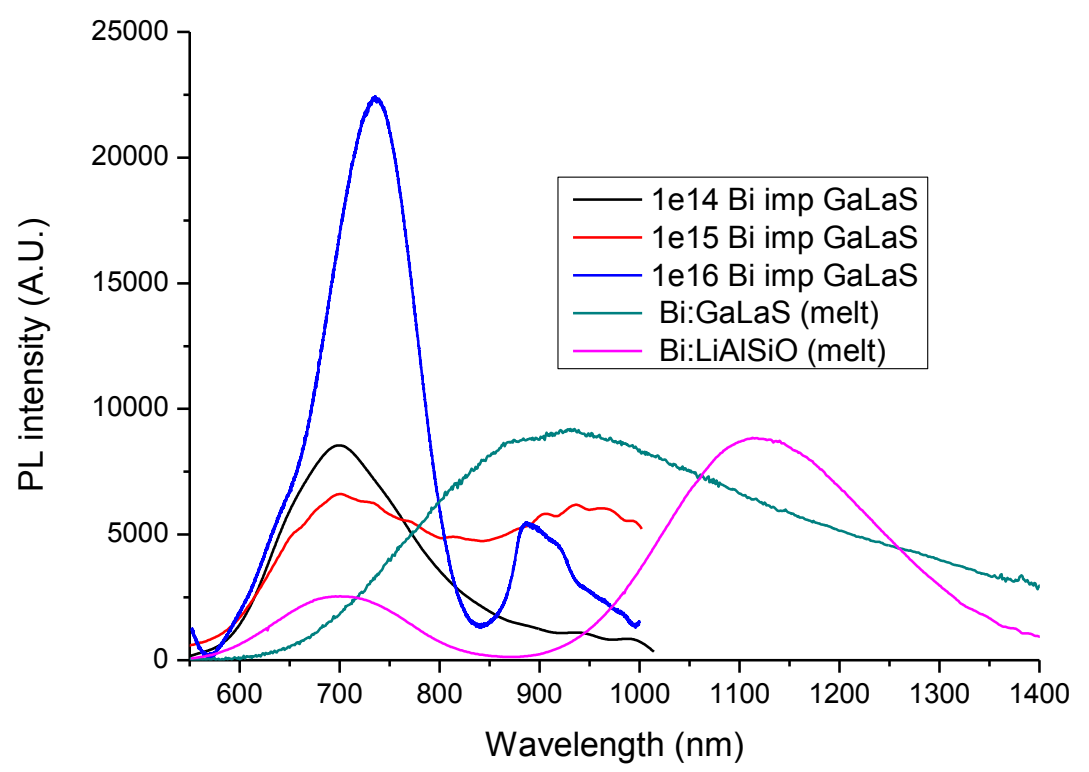

Figure 3 PL spectra of Bi- and Pb-implanted GLSO films at various doses, and bulk Bi-doped GLSO and LiAlSiO glass, excited at $514 \mathrm{~nm}$. The PL intensities of the implanted films are plotted relative to each other, whereas the bulk samples are not.

$\mathrm{Bi}$ - and $\mathrm{Pb}$-doped chalcogenides are the only melt-doped chalcogenide glasses in which carrier-type reversal has been observed. Investigators of this phenomenon have usually fabricated a series of $\mathrm{Bi}$ - or $\mathrm{Pb}$-doped chalcogenide glasses in which the doping content is increased, and measured the thermopower as a function of dopant content. In Bi-doped GeGaS glass, the addition of $\mathrm{Bi}$ caused a red-shift in the absorption edge, rather than the appearance of characteristic Bi absorption bands observed in oxide glasses [11]. In fact, the addition of just 0.15 at.\% Bi caused a red-shift in the band edge of around $100 \mathrm{~nm}$. A similar red-shift is observed when $\mathrm{Bi}$ is added to $\mathrm{GeB}(\mathrm{B}=\mathrm{S}, \mathrm{Se}, \mathrm{Te})$ glasses in which CTR is observed [42], and this red-shift is associated with the CTR. Figure 4(a) shows characteristic Bi-related PL from a Bi-doped GeGaS glass 
$\left(\mathrm{Ge}_{23} \mathrm{Ga}_{12} \mathrm{~S}_{64} \mathrm{Bi}_{1}\right)$ [11]; at lower $\mathrm{Bi}$ concentrations, the PL peak is very similar to a Bi-doped germanate glass. With increasing Bi concentration, the PL becomes significantly weaker and shifts to longer wavelengths. This is probably related to the large red-shift in band edge caused by the addition of $\mathrm{Bi}$. The composition of this glass is very similar to those of glasses in which CTR has been observed $\left(\mathrm{Ge}_{20} \mathrm{Sb}_{11} \mathrm{~S}_{65} \mathrm{Bi}_{4}\right.$ and $\left.\mathrm{Ge}_{20} \mathrm{~S}_{73} \mathrm{Bi}_{7}\right)$, as shown in Figure 4(b). It is therefore reasonable to assume that similar Bi centers are generated in glasses displaying CTR and PL. Since PL in Bi-doped glasses is widely thought to originate from multiple Bi centers, it is likely that one or more of these centers causes the CTR. The observation of NIR PL would suggest these centers are Bi clusters [40].
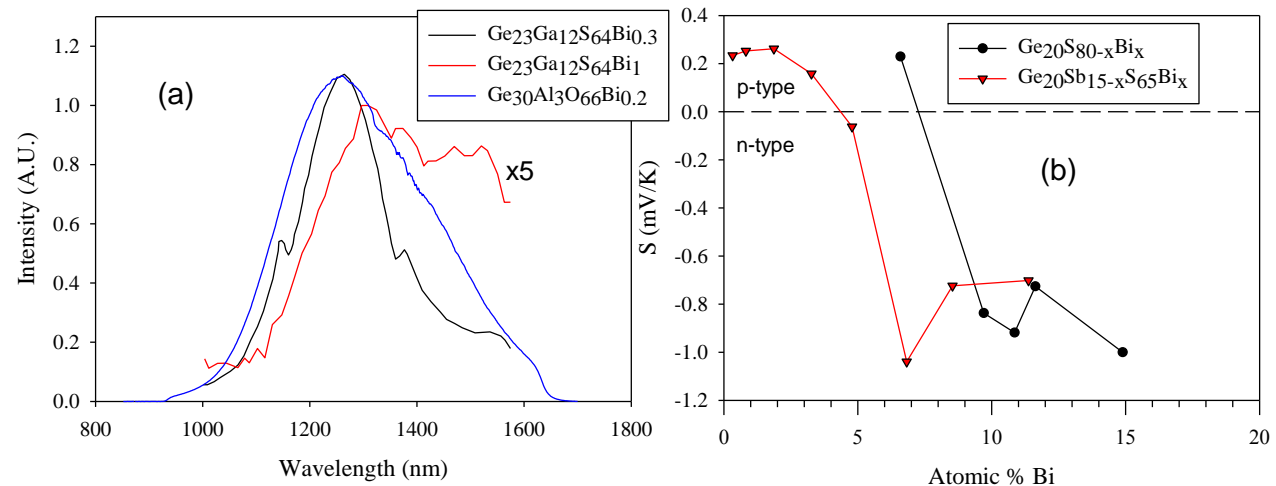

Figure 4 (a) PL spectra of Bi-doped GeGaS, after [11], and Bi-doped germanate glass, with $808 \mathrm{~nm}$ excitation. (b) Thermopower as a function of Bi content in GeS glass, after [26], and in GeSbS glass, after [43] .

\section{Conclusions}

Comparing contour plots of PL spectra at various excitation wavelengths of Bi-doped chalcogenide, Bi-doped germanate and $\mathrm{Pb}$-doped germanate glasses, indicates that five absorption/PL bands are in approximately the same position. This suggests that very similar active centers are present in $\mathrm{Bi}$ - and $\mathrm{Pb}$-doped oxide and chalcogenide glasses. When excited at $782 \mathrm{~nm}, \mathrm{Bi}$-implanted GaLaSO thin films, along with bulk phosphate and silica glass, and $\mathrm{BaF}_{2}$ crystal all display characteristic narrow PL bands peaking between 800 and $880 \mathrm{~nm}$, whereas when melt-doped, these materials display a broad PL peaking between 1100 and $1300 \mathrm{~nm}$. When excited at $514 \mathrm{~nm}$, Bi-implanted GaLaSO thin films display a PL band at $700 \mathrm{~nm}$, which is not present in a Bi melt-doped chalcogenide glass having a similar composition to the implanted glass. This indicates that new $\mathrm{Bi}$ centers are formed through implantation, which are absent in the melt-doped glasses. This has important implications for Bi-doped glass lasers, in which the control of Bi centers is critical for improving performance. We highlight NIR PL bands in $\mathrm{Ge}_{23} \mathrm{Ga}_{12} \mathrm{~S}_{64} \mathrm{Bi}_{1}$ glass, which has a very similar composition to those in which carrier-type reversal has been observed. This indicates that Bi-related PL and carrier-type reversal may be caused by the same $\mathrm{Bi}$ centers, which we suggest are interstitial $\mathrm{Bi}^{2+}$ and $\mathrm{Bi}$ clusters.

\section{Acknowledgments}

This work was supported by the UK EPSRC grants EP/I018414/1, EP/I019065/1 and EP/I018050/1.

\section{REFERENCES}

1. G. W. Chi, D. C. Zhou, Z. G. Song, and J. B. Qiu, "Effect of optical basicity on broadband infrared fluorescence in bismuth-doped alkali metal germanate glasses," Opt. Mater. 31, 945-948 (2009). 
2. A. N. Romanov, Z. T. Fattakhova, A. A. Veber, O. V. Usovich, E. V. Haula, V. N. Korchak, V. B. Tsvetkov, L. A. Trusov, P. E. Kazin, and V. B. Sulimov, "On the origin of near-IR luminescence in Bi-doped materials (II). Subvalent monocation $\mathrm{Bi}^{+}$and cluster $\mathrm{Bi}_{5}{ }^{3+}$ luminescence in $\mathrm{AlCl}_{3} / \mathrm{ZnCl}_{2} / \mathrm{BiCl}_{3}$ chloride glass," Opt. Express 20, 7212-7220 (2012).

3. M. Peng, D. Chen, J. Qiu, X. Jiang, and C. Zhu, "Bismuth-doped zinc aluminosilicate glasses and glassceramics with ultra-broadband infrared luminescence," Opt. Mater. 29, 556-561 (2007).

4. Y. Arai, T. Suzuki, Y. Ohishi, S. Morimoto, and S. Khonthon, "Ultrabroadband near-infrared emission from a colorless bismuth-doped glass," Appl. Phys. Lett. 90, 261110 (2007).

5. M. Peng, B. Wu, N. Da, C. Wang, D. Chen, C. Zhu, and J. Qiu, "Bismuth-activated luminescent materials for broadband optical amplifier in WDM system," J. Non-Cryst. Solids 354, 1221-1225 (2008).

6. J. Ren, J. Qiu, B. Wu, and D. Chen, "Ultrabroad infrared luminescence from Bi-doped alkaline earth metal germanate glasses," J. Mater. Res. 22, 1574-1577 (2007).

7. M. Peng, J. Qiu, D. Chen, X. Meng, I. Yang, X. Jiang, and C. Zhu, "Bismuth and aluminium codoped germanium oxide glasses for super-broadband optical amplification," Opt. Lett. 29, 1998-2000 (2004).

8. M. Peng, J. Qiu, D. Chen, X. Meng, and C. Zhu, "Superbroadband $1310 \mathrm{~nm}$ emission from bismuth and tantalum codoped germanium oxide glasses," Opt. Lett. 30, 2433-2435 (2005).

9. X. G. Meng, J. R. Qiu, M. Y. Peng, D. P. Chen, Q. Z. Zhao, X. W. Jiang, and C. S. Zhu, "Near infrared broadband emission of bismuth-doped aluminophosphate glass," Opt. Express 13, 1628-1634 (2005).

10. X. G. Meng, J. R. Qiu, M. Y. Peng, D. P. Chen, Q. Z. Zhao, X. W. Jiang, and C. S. Zhu, "Infrared broadband emission of bismuth-doped barium-aluminum-borate glasses," Opt. Express 13, 1635-1642 (2005).

11. G. P. Dong, X. D. Xiao, J. J. Ren, J. Ruan, X. F. Liu, J. R. Qiu, C. G. Lin, H. Z. Tao, and X. J. Zhao, "Broadband infrared luminescence from bismuth-doped $\mathrm{GeS}_{2}-\mathrm{Ga}_{2} \mathrm{~S}_{3}$ chalcogenide glasses," Chin. Phys. Lett. 25, 1891-1894 (2008).

12. M. A. Hughes, T. Akada, T. Suzuki, Y. Ohishi, and D. W. Hewak, "Ultrabroad emission from a bismuth doped chalcogenide glass," Opt. Express 17, 19345-19355 (2009).

13. Y. Fujimoto and M. Nakatsuka, "Infrared Luminescence from Bismuth-Doped Silica Glass," Jpn. J. Appl. Phys. Part 2 Lett. 40, L279-L281 (2001).

14. X. Wang and H. Xia, "Infrared superbroadband emission of Bi ion doped germanium-aluminum-sodium glass," Opt. Commun. 268, 75-78 (2006).

15. M. Y. Sharonov, A. B. Bykov, V. Petricevic, and R. R. Alfano, "Spectroscopic study of optical centers formed in $\mathrm{Bi}-, \mathrm{Pb}-, \mathrm{Sb}-, \mathrm{Sn}-$, Te-, and In-doped germanate glasses," Opt. Lett. 33, 2131-2133 (2008).

16. S. Khonthon, S. Morimoto, Y. Arai, and Y. Ohishi, "Luminescence Characteristics of Te- and Bi-Doped Glasses and Glass-Ceramics," J. Ceram. Soc. Jpn. 115, 259-263 (2007).

17. V. O. Sokolov, V. G. Plotnichenko, and E. M. Dianov, "Origin of broadband near-infrared luminescence in bismuth-doped glasses," Opt. Lett. 33, 1488-1490 (2008).

18. I. A. Bufetov and E. M. Dianov, "Bi-doped fiber lasers," Laser Phys. Lett. 6, 487-504 (2009).

19. S. V. Firstov, A. V. Shubin, V. F. Khopin, M. A. Mel'kumov, I. A. Bufetov, O. I. Medvedkov, A. N. Guryanov, and E. M. Dianov, "Bismuth-doped germanosilicate fibre laser with 20-W output power at $1460 \mathrm{~nm}$," Quantum Electron. 41, 581 (2011).

20. V. V. Dvoyrin, V. M. Mashinsky, and E. M. Dianov, "Efficient Bismuth-Doped Fiber Lasers," IEEE J. Quantum Elect. 44, 834-840 (2008).

21. S. Kivisto, J. Puustinen, M. Guina, O. G. Okhotnikov, and E. M. Dianov, "Tunable modelocked bismuth-doped soliton fibre laser," Electron. Lett. 44, 1456-1458 (2008).

22. A. B. Rulkov, A. A. Ferin, S. V. Popov, J. R. Taylor, I. Razdobreev, L. Bigot, and G. Bouwmans, "Narrow-line, $1178 \mathrm{~nm}$ CW bismuth-doped fiber laser with 6.4W output for direct frequency doubling," Opt. Express 15, 5473-5476 (2007).

23. S. Yoo, M. P. Kalita, J. Sahu, J. Nilsson, and D. Payne, "Bismuth-doped fiber laser at $1.16 \mathrm{~mm}$," in Lasers and Electro-Optics, Conference on Quantum Electronics and Laser Science. CLEO/QELS, 2008), 1-2.

24. J. C. Phillips, "Constraint theory and carrier-type reversal in Bi-Ge chalcogenide alloy glasses," Phys. Rev. B 36, 4265-4270 (1987).

25. K. L. Bhatia, D. P. Gosain, G. Parthasarathy, and E. S. R. Gopal, "On the structural features of doped amorphous chalcogenide semiconductors," J. Non-Cryst. Solids 86, 65-71 (1986).

26. L. Tichý, H. Tichá, A. Třiska, and P. Nagels, "Is the n-type conductivity in some Bi-doped chalcogenide glasses controlled by percolation?," Solid State Commun. 53, 399-402 (1985). 
27. V. K. Bhatnagar and K. L. Bhatia, "Frequency dependent electrical transport in bismuth-modified amorphous germanium sulfide semiconductors," J. Non-Cryst. Solids 119, 214-231 (1990).

28. S. R. Elliott and A. T. Steel, "Mechanism for Doping in Bi Chalcogenide Glasses," Phys. Rev. Lett. 57, 13161319 (1986).

29. P. Kounavis, E. Mytilineou, and M. Roilos, "p-n junctions from sputtered $\mathrm{Ge}_{25} \mathrm{Se}_{75-\mathrm{x}} \mathrm{Bi}$, films," J. App. Phys. 66, 708-710 (1989).

30. H. Fritzsche and M. Kastner, "The effect of charged additives on the carrier concentrations in lone-pair semiconductors," Philos. Mag. B 37, 285-292 (1978).

31. S. Okano, H. Yamakawa, M. Suzuki, and A. Hiraki, "Fabrication of Chalcogenide Amorphous Semiconductor Diodes Using Low Temperature Thermal Diffusion Techniques," Jpn. J. Appl. Phys. 26, 1102-1106 (1987).

32. S. Okano, M. Suzuki, T. Imura, and A. Hiraki, "Chalcogenide amorphous-semiconductor diodes," Jpn. J. Appl. Phys. Part 2 Lett. 24, L445-L448 (1985).

33. S. Okano and M. Suzuki, "Electrical contact properties of metal-chalcogenide amorphous-semiconductor systems," Jpn. J. Appl. Phys. 20, 1635-1640 (1981).

34. T. Suzuki and Y. Ohishi, "Ultrabroadband near-infrared emission from Bi-doped $\mathrm{Li}_{2} \mathrm{O}-\mathrm{Al}_{2} \mathrm{O}_{3}-\mathrm{SiO}_{2}$ glass," Appl. Phys. Lett. 88, 191912 (2006).

35. S. Parke and R. S. Webb, "The optical properties of thallium, lead and bismuth in oxide glasses," J. Phys. Chem. Solids 34, 85 (1973).

36. X. G. Meng, J. R. Qiu, M. Y. Peng, D. P. Chen, Q. Z. Zhao, X. W. Jiang, and C. S. Zhu, "Near infrared broadband emission of bismuth-doped aluminophosphate glass," Opt. Express 13, 1628-1634 (2005).

37. Y. Fujimoto and M. Nakatsuka, "Infrared Luminescence from Bismuth-Doped Silica Glass," Jpn. J. Appl. Phys., Part 2 40, L279-L281 (2001).

38. J. Ruan, L. B. Su, J. R. Qiu, D. P. Chen, and J. Xu, "Bi-doped BaF 2 crystal for broadband near-infrared light source," Opt. Express 17, 5163-5169 (2009).

39. B. I. Denker, B. I. Galagan, V. V. Osiko, I. L. Shulman, S. E. Sverchkov, and E. M. Dianov, "Factors affecting the formation of near infrared-emitting optical centers in Bi-doped glasses," Appl. Phys. B-Lasers Opt. 98, 455458 (2010).

40. M. A. Hughes, R. M. Gwilliam, K. Homewood, B. Gholipour, D. W. Hewak, T.-H. Lee, S. R. Elliott, T. Suzuki, Y. Ohishi, T. Kohoutek, and R. J. Curry, "On the analogy between photoluminescence and carrier-type reversal in $\mathrm{Bi}$ - and Pb-doped glasses " Opt. Express 21, 8101-8115 (2013).

41. Y. Kim, J. H. Baeck, M.-H. Cho, E. J. Jeong, and D.-H. Ko, "Effects of $\mathrm{N}^{2+}$ ion implantation on phase transition in $\mathrm{Ge}_{2} \mathrm{Sb}_{2} \mathrm{Te}_{5}$ films," J. App. Phys. 100, 083502 (2006).

42. N. Tohge, T. Minami, Y. Yamamoto, and M. Tanaka, "Electrical and optical properties of n-type semiconducting chalcogenide glasses in the system Ge-Bi-Se," J. App. Phys. 51, 1048-1053 (1980).

43. J. Málek, J. Klikorka, L. Beneš, L. Tichý, and A. Tříska, "Electrical and optical properties of $\mathrm{Ge}_{20} \mathrm{Sb}_{15-\mathrm{x}} \mathrm{Bi}_{\mathrm{x}} \mathrm{Bi}_{65}$ glasses," J. Mater. Sci. 21, 488-492 (1986). 